\title{
Robust dual topological character with spin-valley polarization in a monolayer of the Dirac semimetal $\mathrm{Na}_{3} \mathrm{Bi}$
}

\author{
Chengwang Niu, ${ }^{1, *}$ Patrick M. Buhl, ${ }^{1}$ Gustav Bihlmayer, ${ }^{1}$ Daniel Wortmann, ${ }^{1}$ Ying Dai, ${ }^{2}$ \\ Stefan Blügel, ${ }^{1}$ and Yuriy Mokrousov ${ }^{1}$ \\ ${ }^{1}$ Peter Grünberg Institut and Institute for Advanced Simulation, Forschungszentrum Jülich and JARA, 52425 Jülich, Germany \\ ${ }^{2}$ School of Physics, State Key Laboratory of Crystal Materials, Shandong University, 250100 Jinan, People's Republic of China
}

(Received 4 November 2016; published 6 February 2017)

\begin{abstract}
Topological materials with both insulating and semimetal phases can be protected by crystalline (e.g., mirror) symmetry. The insulating phase, called a topological crystalline insulator (TCI), has been investigated intensively and observed in three-dimensional materials. However, the predicted two-dimensional (2D) materials with TCI phase are explored much less than 3D TCIs and 2D topological insulators, while the 2D TCIs considered thus far possess almost exclusively a square-lattice structure with the mirror Chern number $\mathcal{C}_{M}=-2$. Here, we predict theoretically that a hexagonal monolayer of Dirac semimetal $\mathrm{Na}_{3} \mathrm{Bi}$ is a $2 \mathrm{D}$ TCI with a mirror Chern number $\mathcal{C}_{M}=-1$. The large nontrivial gap of $0.31 \mathrm{eV}$ is tunable and can be made much larger via strain engineering, while the topological phases are robust against strain, indicating a high possibility for room-temperature observation of quantized conductance. In addition, a nonzero spin Chern number $\mathcal{C}_{S}=-1$ is obtained, indicating the coexistence of a 2D topological insulator and a 2D TCI, i.e., the dual topological character. Remarkably, a spin-valley polarization is revealed in the $\mathrm{Na}_{3} \mathrm{Bi}$ monolayer due to the breaking of crystal inversion symmetry. The dual topological character is further explicitly confirmed via the unusual behavior of the edge states under the corresponding symmetry breaking.
\end{abstract}

DOI: 10.1103/PhysRevB.95.075404

The discovery of topological insulators (TIs) [1,2] has triggered an explosion of novel topologically nontrivial phases, such as the topological crystalline insulator (TCI), for which the role of time-reversal symmetry is replaced by crystal (mirror) symmetry [3-5]. The hallmark of a TCI, similar to a TI, is the presence of gapless surface/edge states with Dirac points inside of the insulating bulk energy gap. In the presence of crystal mirror symmetry, the coexistence of TI and TCI phases has been predicted in three dimensions for $\mathrm{Bi}_{1-x} \mathrm{Sb}_{x}$ [6] and Bi chalcogenides [7-9], and thus they exhibit a dual topological character (DTC). Recently, unusual topological surface states for a three-dimensional (3D) DTC system have been observed experimentally [8,9]. In the 2D case, graphene may be a prototypical example of a DTC $[10,11]$. However, the extremely small band gap of graphene makes it very difficult to verify the DTC in this material experimentally [12]. To date, 2D TIs have been identified experimentally in $\mathrm{HgTe} / \mathrm{CdTe}$ [13] and InAs/GaSb [14] quantum wells at low temperatures, and many 2D TIs with giant band gaps have been predicted to exist as a result of the substrate interaction effect [15], chemical functionalization [16-20], or global structure optimization [21]. In many cases, the complex structures and the lack of mirror symmetry in such materials prevent the formation of a 2D TCI phase. On the other hand, 2D TCIs have been limited thus far to theoretical predictions that are mainly restricted to SnTe multilayers [10], $(\mathrm{Sn} / \mathrm{Pb})(\mathrm{Se} / \mathrm{Te})$ monolayers [22-24], Tl(S/Se) monolayers [25], and $\mathrm{SnTe} / \mathrm{NaCl}$ quantum wells [26] with mirror Chern number $\mathcal{C}_{M}=-2$. The even number of band inversions leads to a vanishing $\mathbb{Z}_{2}$ invariant. Therefore, 2 D TCIs with $\mathcal{C}_{M}=-2$ cannot be $2 \mathrm{D}$ TIs protected by time-reversal symmetry. Thus, for the continued investigation and additional applications

*c.niu@fz-juelich.de of a DTC in two dimensions, it is essential to extend the domain of candidate 2D TIs and TCIs with respect to both topological manifestations (i.e., different $\mathcal{C}_{M}$ [27]) and material realization.

For both 2D TIs and 2D TCIs, spin-orbit coupling (SOC) is known to play a vital role. In addition, SOC together with inversion symmetry breaking can lead to coupled spin and valley physics, in which the new degree of freedom offers a promising route to the eventual realization of valleytronic devices [28,29]. Spin-valley polarization has been observed experimentally in the $\mathrm{MoS}_{2}$ monolayer [30], which is a topologically trivial insulator. Therefore, a natural question arises as to whether spin-valley polarization in nontrivial insulators, such as 2D TIs and 2D TCIs, is possible. Recently, thin films of the Dirac semimetal $\mathrm{Na}_{3} \mathrm{Bi}[31,32]$ were fabricated by molecular-beam epitaxy [33], and therefore in the present study we take $\mathrm{Na}_{3} \mathrm{Bi}$ as an example and propose the realization of the 2D DTC in a monolayer of $\mathrm{Na}_{3} \mathrm{Bi}$ with a band gap of 0.31 $\mathrm{eV}$, which is well above the energy scale of room temperature. The calculated spin Chern number $\mathcal{C}_{S}=-1$ and the mirror Chern number $\mathcal{C}_{M}=-1$ confirm the 2D DTC phase directly. In addition, spin-valley polarization due to a lack of spatial inversion symmetry is investigated.

Density-functional calculations are performed using the generalized-gradient approximation (GGA) of Perdew-BurkeErnzerhof (PBE) [34] for the exchange correlation potential as implemented in the FLEUR code [35] as well as in the Vienna ab-initio simulation package (VASP) [36,37]. A $20 \AA$ thick vacuum layer is used to avoid interactions between nearest slabs for VASP, while the film calculations are carried out with the film version of the FLEUR code [38]. SOC is included in the calculations self-consistently. The maximally localized Wannier functions (MLWFs) are constructed using the WANNIER90 code in conjunction with the FLEUR package $[39,40]$. 


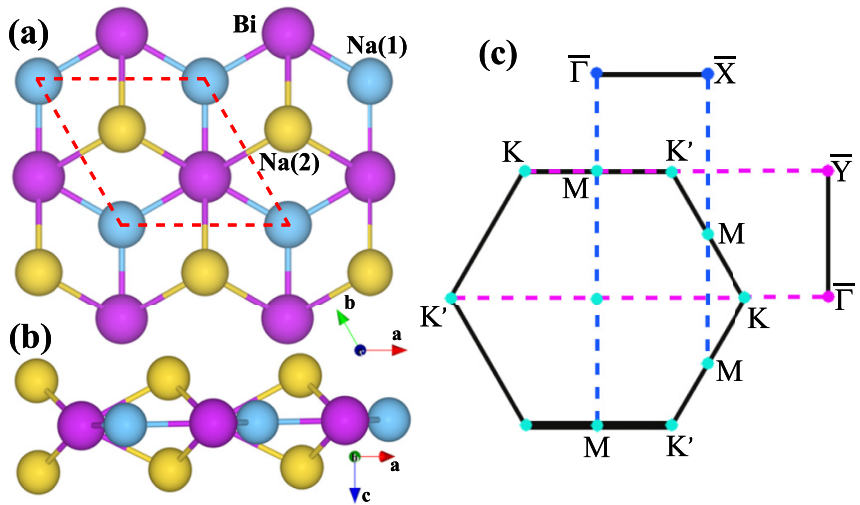

FIG. 1. (a) Top and (b) side view of the honeycomb $\mathrm{Na}_{3} \mathrm{Bi}$ monolayer, where the unit cell is indicated by the dashed lines. (c) The Brillouin zone of the $2 \mathrm{D} \mathrm{Na} 3 \mathrm{Bi}$ monolayer and the projected $1 \mathrm{D}$ Brillouin zones.

Bulk $\mathrm{Na}_{3} \mathrm{Bi}$ in a hexagonal $P 6_{3} / m m c$ structure is a $3 \mathrm{D}$ counterpart of graphene, which hosts 3D Dirac points in its electronic structure and is called a topological Dirac semimetal [31,32]. The bulk crystal structure consists of stacked triple layers along the $z$ direction. Each triple layer has four atoms, i.e., one $\mathrm{Bi}$ in the Wyckoff $2 c$ position, one $\mathrm{Na}(1)$ in the $2 b$ position, and two $\mathrm{Na}(2)$ in the $4 f$ position. In Figs. 1(a) and 1(b), the side and top view of the $\mathrm{Na}_{3} \mathrm{Bi}$ triple layer are presented, with $\mathrm{Bi}$ and $\mathrm{Na}$ atoms forming a honeycomb lattice. Unlike in the bulk material, inversion symmetry is broken in a $\mathrm{Na}_{3} \mathrm{Bi}$ triple layer, but the mirror symmetry $z \rightarrow-z$ is preserved, in exact analogy to a $\mathrm{MoS}_{2}$ monolayer [41]. Hereafter, we call such a triple layer a $\mathrm{Na}_{3} \mathrm{Bi}$ monolayer. To check its energetic stability, the formation energy is calculated by $E_{f}=E_{\mathrm{Na}_{3} \mathrm{Bi}}-3 \mu_{\mathrm{Na}}-\mu_{\mathrm{Bi}}$, where $\mathrm{E}_{\mathrm{Na}_{3} \mathrm{Bi}}$ is the total energy of the $\mathrm{Na}_{3} \mathrm{Bi}$ monolayer, and $\mu_{\mathrm{Na}}$ and $\mu_{\mathrm{Bi}}$ are the chemical potentials of $\mathrm{Na}$ and $\mathrm{Bi}$ atoms, respectively. The calculated formation energy of $-0.78 \mathrm{eV}$ indicates that the $\mathrm{Na}_{3} \mathrm{Bi}$ monolayer is energetically stable.

Figures 2(a) and 2(b) present the orbitally resolved band structures of the $\mathrm{Na}_{3} \mathrm{Bi}$ monolayer without and with $\mathrm{SOC}$, respectively, which deliver preliminary insight into the topological properties of the system. Due to the presence of time-reversal symmetry, the bands at valleys $K$ and $K^{\prime}$ are energetically degenerate, and thus we only show the dispersion around $K$. In the absence of $\mathrm{SOC}, \mathrm{Bi}-p_{x}$ and $\mathrm{Bi}-p_{y}$ orbitals contribute to the valence-band maximum (VBM), while the conduction-band minimum (CBM) is dominated by $\mathrm{Bi}-s$ orbitals with a direct band gap of $0.16 \mathrm{eV}$. Switching on SOC leads to an inversion of the VBM and the CBM, and an $s$ - $p$ band inversion occurs at the $\Gamma$ point. The insulating character is preserved with a band gap of $0.31 \mathrm{eV}$, indicating the feasibility of an experimental observation of the 2D topological properties of this material at room temperature. To further confirm our results, the band structure is checked by using the more sophisticated Heyd-Scuseria-Ernzerhof hybrid functional method (HSE06) [42]. Similar to $1-T^{\prime} \mathrm{MoS}_{2}$ [43], the nontrivial phase has a larger band gap $(\sim 0.4 \mathrm{eV})$ when SOC is included.

The existence of the mirror symmetry $z \rightarrow-z$ [see Figs. 1(a) and 1(b)] for the $\mathrm{Na}_{3} \mathrm{Bi}$ monolayer offers the
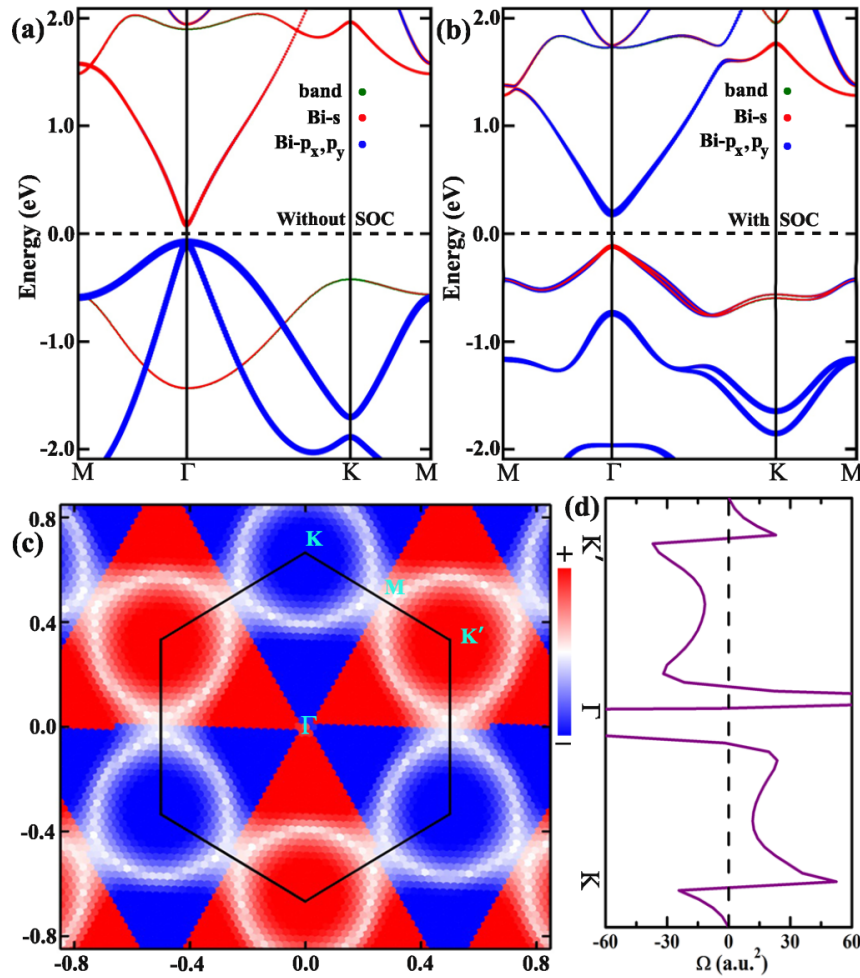

FIG. 2. Orbitally resolved band structures for the $\mathrm{Na}_{3} \mathrm{Bi}$ monolayer (a) without and (b) with SOC, weighted with the contribution of $\mathrm{Bi}-s$ and $\mathrm{Bi}-p_{x}, p_{y}$ states. The Fermi level is indicated with a dashed line. (c) Momentum-resolved polarization of spin perpendicular to the mirror plane for the highest occupied band. (d) Berry curvature distribution of the highest occupied bands in the $K-\Gamma-K^{\prime}$ direction.

possibility of realizing a TCI that is characterized by the so-called mirror Chern number [3,6], which is defined as $\mathcal{C}_{M}=$ $\left(\mathcal{C}_{+i}-\mathcal{C}_{-i}\right) / 2$, where $\mathcal{C}_{+i}$ and $\mathcal{C}_{-i}$ are the Chern numbers for mirror eigenvalues $+i$ and $-i$ [44],

$$
\mathcal{C}_{ \pm i}=\frac{1}{2 \pi} \sum_{n<E_{F}} \int_{\mathrm{BZ}} \Omega_{ \pm i}(\mathbf{k}) d^{2} k
$$

and $\Omega_{ \pm i}(\mathbf{k})$ is the Berry curvature of all occupied bands constructed from respective mirror projected states in the mirror plane, calculated according to

$$
\Omega(\mathbf{k})=-2 \operatorname{Im} \sum_{m \neq n} \frac{\left\langle\psi_{n \mathbf{k}}\left|v_{x}\right| \psi_{m \mathbf{k}}\right\rangle\left\langle\psi_{m \mathbf{k}}\left|v_{y}\right| \psi_{n \mathbf{k}}\right\rangle}{\left(\varepsilon_{m \mathbf{k}}-\varepsilon_{n \mathbf{k}}\right)^{2}}
$$

where $m, n$ are band indices, $\psi_{m / n \mathbf{k}}$ and $\varepsilon_{m / n \mathbf{k}}$ are the corresponding wave functions and eigenenergies of band $m / n$, respectively, and $v_{x / y}$ are the velocity operators. The MLWFs are constructed to calculate the Berry curvature efficiently. With $z \rightarrow-z$ mirror symmetry, the calculated Chern numbers are, respectively, $\mathcal{C}_{ \pm i}=\mp 1$, leading to a mirror Chern number $\mathcal{C}_{M}=-1$. This indicates that the $\mathrm{Na}_{3} \mathrm{Bi}$ monolayer is a 2D TCI. Interestingly, the $\mathcal{C}_{M}=-1$ case we consider here is topologically distinct from the previously reported 2D TCIs, such as $(\mathrm{Sn} / \mathrm{Pb}) \mathrm{Te}[10,23,24]$ and $\mathrm{Tl}(\mathrm{S} / \mathrm{Se})[25]$ with $\mathcal{C}_{M}=-2$.

Here, in the $\mathrm{Na}_{3} \mathrm{Bi}$ monolayer, band inversion occurs at the $\Gamma$ point, i.e., we acquire an odd number of band inversions. To 

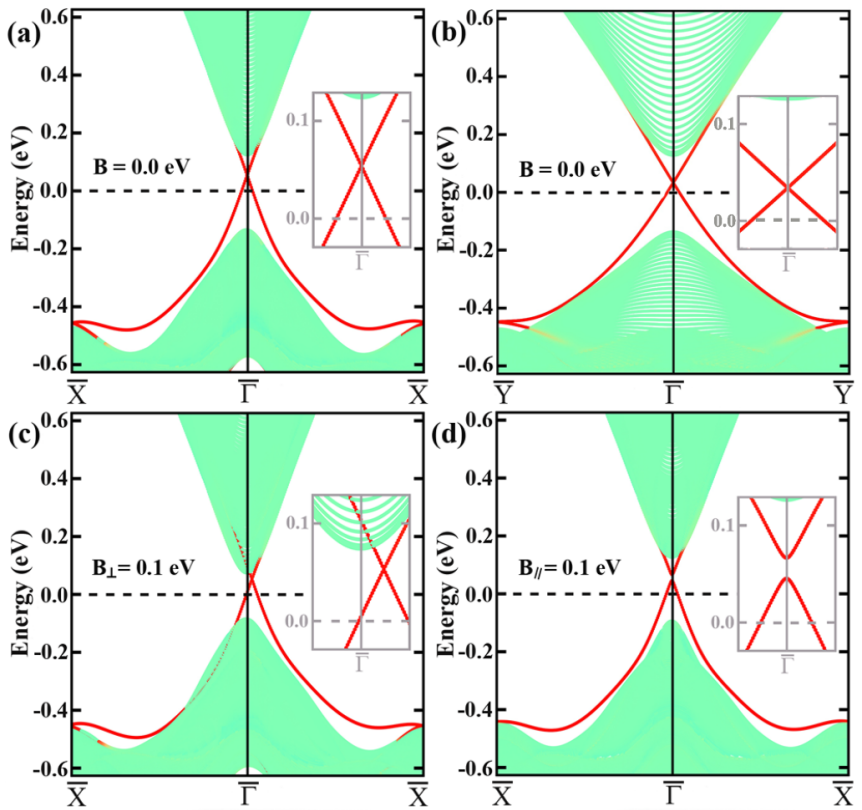

FIG. 3. Localization-resolved edge states of the $\mathrm{Na}_{3} \mathrm{Bi}$ monolayer for different configurations. (a) $\mathrm{Bi}-\mathrm{Na}(1)$ termination without a magnetic field, (b) $\mathrm{Bi}-\mathrm{Na}(1)-\mathrm{Na}(2)$ termination without a magnetic field, (c) $\mathrm{Bi}-\mathrm{Na}(1)$ termination with a magnetic field perpendicular to the mirror plane, and (d) $\mathrm{Bi}-\mathrm{Na}(1)$ termination with a magnetic field within the mirror plane. The insets show the corresponding zoom-in at the $\bar{\Gamma}$ point. Color from light green to red represents the weight of atoms located from the middle to one edge of the ribbon structures.

identify the relationship between the 2D TI and the odd number of band inversions in the $\mathrm{Na}_{3} \mathrm{Bi}$ monolayer, we calculate the spin Chern number $\mathcal{C}_{S}$ [45-47], which can be directly related to the $\mathbb{Z}_{2}$ topological invariant of the system. $\mathcal{C}_{S}$ provides an equivalent characterization to the $\mathbb{Z}_{2}$ number in that for 2D time-reversal symmetric systems, the even values of $\mathcal{C}_{S}$ correspond to a topologically trivial insulator state, while odd values of $\mathcal{C}_{S}$ indicate the emergence of a TI phase [45-47]. $\mathcal{C}_{S}$ is given by the difference of the Chern numbers for the spin-up $\left(\mathcal{C}_{+}\right)$and spin-down $\left(\mathcal{C}_{-}\right)$projected manifolds, $\mathcal{C}_{S}=$ $\left(\mathcal{C}_{+}-\mathcal{C}_{-}\right) / 2$ [45]. The $\sigma_{z}$ matrix, $\left\langle\phi_{m \mathbf{k}}\left|\sigma_{z}\right| \phi_{n \mathbf{k}}\right\rangle$, is constructed and diagonalized to distinguish the spin-up and spin-down manifolds [47]. The Chern number for each spin manifold is $\mathcal{C}_{+}=-1$ and $\mathcal{C}_{-}=1$, yielding the spin Chern number $\mathcal{C}_{S}=$ -1 . This clearly demonstrates the $2 \mathrm{D}$ TI nature of the $\mathrm{Na}_{3} \mathrm{Bi}$ monolayer. Therefore, the $\mathrm{Na}_{3} \mathrm{Bi}$ monolayer exhibits the DTC with respect to the $2 \mathrm{D} \mathrm{TI}$ and $2 \mathrm{D}$ TCI phases.

To further confirm the DTC, we investigate the edge states of $1 \mathrm{D}$ nanoribbons of the $\mathrm{Na}_{3} \mathrm{Bi}$ monolayer. Nonzero $\mathcal{C}_{M}$ and/or $\mathcal{C}_{S}$ should support the gapless edge states bridging the conduction and valence bands, which exhibit a band gap when both time-reversal and mirror symmetries are broken. Based on a description in terms of MLWFs of the $\mathrm{Na}_{3} \mathrm{Bi}$ monolayer, the tight-binding Hamiltonians of nanoribbons along two different directions with a width of 60 unit cells are constructed. The calculated band structures on one side of the ribbons are presented in Figs. 3(a) and 3(b), respectively. One can clearly see a pair of gapless edge states in the $2 \mathrm{D}$ gap. The Dirac point around $\bar{\Gamma}$ is a clear consequence of the nontrivial topological character of the system.

Generally, time-reversal symmetry breaking generates a gap in the surface/edge states of TIs [1,2], while mirror symmetry breaking is indispensable for the formation of a band gap in the surface/edge states of TCIs [3]. One way to destroy these symmetries is to introduce magnetism in the system. To mimic a magnetic environment, we compute the matrix elements of the Pauli matrices $\sigma_{\alpha}(\alpha=x, y, z)$ on the basis of MLWFs, which allows us to consider the effect of an exchange field applied along different directions. For an exchange field perpendicular to the mirror plane, $H_{\text {mag }}=B_{\perp} \sigma_{z}$, time-reversal symmetry is broken while mirror symmetry is maintained. In this case, as shown in Fig. 3(c) for the Bi-Na(1) termination, the Dirac point moves slightly away from the $\bar{\Gamma}$ point, while a band gap does not open as a consequence of the 2D TCI phase's survival. If the exchange field, on the other hand, is in the plane, $H_{\text {mag }}=B_{\|} \sigma_{x}$, both time-reversal and mirror symmetries are broken and the edge states become gapped [Fig. 3(d)]. This behavior is reminiscent of that in $\mathrm{Bi}_{2}(\mathrm{Se} / \mathrm{Te})_{3}$ $[7,48]$, but with different directions of an exchange field due to a different sense of the mirror symmetry in these two compounds ( $B_{z}$ in the $3 \mathrm{D}$ TI corresponds to $B_{\|}$in the $2 \mathrm{D} \mathrm{TI}$ ) $[7,48]$.

Exposing honeycomb lattices to inversion symmetry breaking provides a new, so-called valley, tunable degree of freedom in addition to spin and charge. The valley degree of freedom is receiving considerable attention at present due to potential application in valleytronics $[28,30]$. To demonstrate the effect of inversion symmetry breaking, we focus now on the spinvalley coupling of the $\mathrm{Na}_{3} \mathrm{Bi}$ monolayer by considering the spin polarization of occupied states in reciprocal space. Since the in-plane components of the spin polarization are vanishing due to the presence of mirror symmetry, in Fig. 2(c) we plot the momentum-resolved out-of-plane spin polarization of the highest occupied band. It is clearly visible that the highest occupied state exhibits spin polarization that is of opposite sign at valleys $K$ and $K^{\prime}$. We further inspect the Berry curvature of the highest occupied band along the $K-\Gamma-K^{\prime}$ path, plotted in Fig. 2(d). An odd behavior of the Berry curvature with respect to the valley agrees with the symmetry analysis in terms of time- and structural-inversion symmetry, similarly to the well-known case of spin-valley coupling in the $\mathrm{MoS}_{2}$ monolayer [49]. Valley polarization that is coupled with spin will suppress spin and valley relaxation and is a promising way to prepare information carriers for next-generation electronic and optoelectronic devices [30].

Having established our material's DTC, accompanied by a band gap of $0.31 \mathrm{eV}$ (which is large enough for practical applications at room temperature), we finally test its stability. The phonon spectrum calculation shows imaginary frequencies around the $M$ point, but not at the $\Gamma$ point, indicating that the band inversion is robust. We demonstrate this by investigating the band inversion under various strains as well as substrates [50]. The magnitude of strain is described by the ratio $a / a_{0}$, where " $a_{0}$ " and " $a$ " denote the lattice parameters of the unstrained (5.31 $\AA$ ) and strained systems, respectively. The results of the calculations shown in Fig. 4 indicate that the band gap of the $\mathrm{Na}_{3} \mathrm{Bi}$ monolayer can be significantly enhanced upon straining, similarly to the results reported previously 

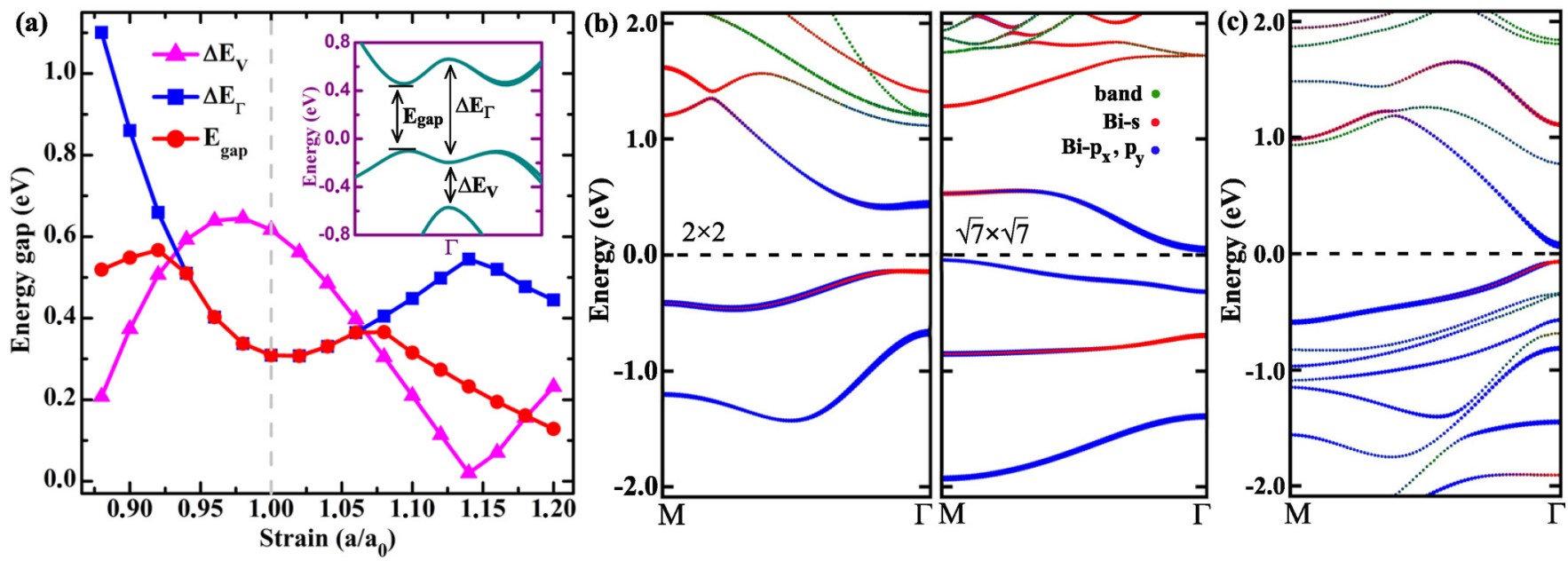

FIG. 4. (a) Variation of the energy gaps for the $\mathrm{Na}_{3} \mathrm{Bi}$ monolayer as a function of strain. The gaps ( $\Delta E_{\Gamma}$ and $E_{\text {gap }}$ in the inset) between the valence and conduction bands remain, while a strain-induced band inversion between the valence bands $\left(\Delta E_{V}\right.$ in the inset) occurs. (b) Orbitally resolved band structures for the $\mathrm{Na}_{3} \mathrm{Bi}$ monolayer sandwiched between a substrate of (b) BN with $2 \times 2$ and $\sqrt{7} \times \sqrt{7}$ supercells and (c) $\mathrm{Na}_{3} \mathrm{Sb}$, weighted with the contribution of $\mathrm{Bi}-s$ and $\mathrm{Bi}-p_{x}, p_{y}$ states. The DTC remains intact with corresponding substrates.

$[18,25]$. There is no band-gap closing-reopening process between the valence and conduction bands $\left(\Delta E_{\Gamma}\right.$ and $\left.E_{\text {gap }}\right)$ in a large range of strain from $-12 \%$ to $20 \%$. The persistent band inversion indicates that the topological character of the system is robust against the substrate-imposed strain, which is important for further experimental investigations and device applications due to the fact that a free-standing film is usually hard to grow. Figure 4(b) shows the calculated band structures of quantum-well structures that retain mirror symmetry, with the $\mathrm{Na}_{3} \mathrm{Bi}$ monolayer sandwiched between $2 \times 2$ and $\sqrt{7} \times$ $\sqrt{7}$ BN monolayers. As we can see, the energy gap at the $M$ point changes much more than at $\Gamma$, with the inverted band gap at the $\Gamma$ point surviving in both cases [although a further band inversion occurs between valence bands for the $\sqrt{7} \times \sqrt{7}$ case; see also $\Delta E_{V}$ of Fig. 4(a)], which proves that the DTC is preserved with a respective substrate-induced strain of $-6.2 \%$ and $19.7 \%$. A weak interlayer interaction is expected between $\mathrm{BN}$ and $\mathrm{Na}_{3} \mathrm{Bi}$ because of the large relaxed interlayer distances $\sim 4.0 \AA$. We then consider a strong interlayer interaction via the topologically trivial substrate $\mathrm{Na}_{3} \mathrm{Sb}$ that has the same crystal structure as $\mathrm{Na}_{3} \mathrm{Bi}$ but creates $-2 \%$ epitaxial strain.
As shown in Fig. 4(c), the band inversion remains at the $\Gamma$ point.

In summary, based on first-principles calculations, we revealed that the $2 \mathrm{D}$ TI and $2 \mathrm{D}$ TCI phases can coexist in the $\mathrm{Na}_{3} \mathrm{Bi}$ monolayer with a large band gap of $0.31 \mathrm{eV}$. The nonzero spin Chern number and the mirror Chern number, as well as nontrivial topological edge states, confirm the dual topological character clearly. As the mirror symmetry is preserved for an out-of-plane exchange field, the gapless edge states survive but move away from the time-reversal invariant momenta, while a gap opens for in-plane exchange fields. Finally, strain engineering shows that the dual topological character in the $\mathrm{Na}_{3} \mathrm{Bi}$ monolayer is robust in a large range of strain in which the nontrivial band gap can be tuned efficiently.

This work was supported by the Priority Program 1666 of the German Research Foundation (DFG) and the Virtual Institute for Topological Insulators (VITI). We acknowledge computing time on the supercomputers JUQUEEN and JURECA at Jülich Supercomputing Centre and JARA-HPC of RWTH Aachen University.
[1] M. Z. Hasan and C. L. Kane, Rev. Mod. Phys. 82, 3045 (2010).

[2] X.-L. Qi and S.-C. Zhang, Rev. Mod. Phys. 83, 1057 (2011).

[3] L. Fu, Phys. Rev. Lett. 106, 106802 (2011).

[4] T. H. Hsieh, H. Lin, J. Liu, W. Duan, A. Bansil, and L. Fu, Nat. Commun. 3, 982 (2012).

[5] Y. Ando and L. Fu, Annu. Rev. Condens. Matter Phys. 6, 361 (2015).

[6] J. C. Y. Teo, L. Fu, and C. L. Kane, Phys. Rev. B 78, 045426 (2008).

[7] T. Rauch, M. Flieger, J. Henk, I. Mertig, and A. Ernst, Phys. Rev. Lett. 112, 016802 (2014).

[8] A. P. Weber, Q. D. Gibson, H. Ji, A. N. Caruso, A. V. Fedorov, R. J. Cava, and T. Valla, Phys. Rev. Lett. 114, 256401 (2015).
[9] M. Eschbach, M. Lanius, C. Niu, E. Młyńczak, P. Gospodarič, J. Kellner, P. Schüffelgen, M. Gehlmann, S. Döring, E. Neumann, M. Luysberg, G. Mussler, L. Plucinski, M. Morgenstern, D. Grützmacher, G. Bihlmayer, S. Blügel, and C. M. Schneider, arXiv:1604.08886.

[10] J. Liu, T. H. Hsieh, P. Wei, W. Duan, J. Moodera, and L. Fu, Nat. Mater. 13, 178 (2014).

[11] C. L. Kane and E. J. Mele, Phys. Rev. Lett. 95, 226801 (2005).

[12] Y. Yao, F. Ye, X.-L. Qi, S.-C. Zhang, and Z. Fang, Phys. Rev. B 75, 041401 (2007).

[13] M. König, S. Wiedmann, C. Brüne, A. Roth, H. Buhmann, L. Molenkamp, X.-L. Qi, and S.-C. Zhang, Science 318, 766 (2007). 
[14] I. Knez, R. R. Du, and G. Sullivan, Phys. Rev. Lett. 107, 136603 (2011).

[15] M. Zhou, W. Ming, Z. Liu, Z. Wang, P. Li, and F. Liu, Proc. Natl. Acad. Sci. USA 111, 14378 (2014).

[16] Y. Xu, B. H. Yan, H. J. Zhang, J. Wang, G. Xu, P. Z. Tang, W. H. Duan, and S.-C. Zhang, Phys. Rev. Lett. 111, 136804 (2013).

[17] Z. Song, C.-C. Liu, J. Yang, J. Han, B. Fu, M. Ye, Y. Yang, Q. Niu, J. Lu, and Y. Yao, NPG Asia Mater. 6, e147 (2014).

[18] C. Niu, G. Bihlmayer, H. Zhang, D. Wortmann, S. Blügel, and Y. Mokrousov, Phys. Rev. B 91, 041303(R) (2015).

[19] L. Li, X. Zhang, X. Chen, and M. Zhao, Nano. Lett. 15, 1296 (2015).

[20] C. P. Crisostomo, L.-Z. Yao, Z.-Q. Huang, C.-H. Hsu, F.-C. Chuang, H. Lin, M. A. Albao, and A. Bansil, Nano. Lett. 15, 6568 (2015).

[21] W. Luo and H. Xiang, Nano Lett. 15, 3230 (2015).

[22] E. O. Wrasse and T. M. Schmidt, Nano Lett. 14, 5717 (2014).

[23] J. Liu, X. Qian, and L. Fu, Nano Lett. 15, 2657 (2015).

[24] C. Niu, P. M. Buhl, G. Bihlmayer, D. Wortmann, S. Blügel, and Y. Mokrousov, Phys. Rev. B 91, 201401(R) (2015).

[25] C. Niu, P. M. Buhl, G. Bihlmayer, D. Wortmann, S. Blügel, and Y. Mokrousov, Nano Lett. 15, 6071 (2015).

[26] C. Niu, P. M. Buhl, G. Bihlmayer, D. Wortmann, S. Blügel, and Y. Mokrousov, 2D Mater. 3, 025037 (2016).

[27] R. Takahashi and S. Murakami, Phys. Rev. Lett. 107, 166805 (2011).

[28] D. Xiao, W. Yao, and Q. Niu, Phys. Rev. Lett. 99, 236809 (2007).

[29] A. Rycerz, J. Tworzydlo, and C. W. J. Beenakker, Nat. Phys. 3, 172 (2007).

[30] K. F. Mak, K. L. McGill, J. Park, and P. L. McEuen, Science 344, 1489 (2014).

[31] Z. Wang, Y. Sun, X.-Q. Chen, C. Franchini, G. Xu, H. Weng, X. Dai, and Z. Fang, Phys. Rev. B 85, 195320 (2012).

[32] Z. K. Liu, B. Zhou, Y. Zhang, Z. J. Wang, H. M. Weng, D. Prabhakaran, S.- K. Mo, Z. X. Shen, Z. Fang, X. Dai, Z. Hussain, and Y. L. Chen, Science 343, 864 (2014).
[33] J. Hellerstedt, M. T. Edmonds, N. Ramakrishnan, C. Liu, B. Weber, A. Tadich, K. M. O'Donnell, S. Adam, and M. S. Fuhrer, Nano Lett. 16, 3210 (2016).

[34] J. P. Perdew, K. Burke, and M. Ernzerhof, Phys. Rev. Lett. 77, 3865 (1996).

[35] www.flapw.de

[36] G. Kresse and J. Hafner, Phys. Rev. B 47, 558 (1993).

[37] G. Kresse and J. Furthmüller, Phys. Rev. B 54, 11169 (1996).

[38] H. Krakauer, M. Posternak, and A. J. Freeman, Phys. Rev. B 19, 1706 (1979).

[39] A. A. Mostofi, J. R. Yates, Y. S. Lee, I. Souza, D. Vanderbilt, and N. Marzari, Comput. Phys. Commun. 178, 685 (2008).

[40] F. Freimuth, Y. Mokrousov, D. Wortmann, S. Heinze, and S. Blügel, Phys. Rev. B 78, 035120 (2008).

[41] A. Splendiani, L. Sun, Y. Zhang, T. Li, J. Kim, C.-Y. Chim, G. Galli, and F. Wang, Nano Lett. 10, 1271 (2010).

[42] A. V. Krukau, O. A. Vydrov, A. F. Izmaylov, and G. E. Scuseria, J. Chem. Phys. 125, 224106 (2006).

[43] X. Qian, J. Liu, L. Fu, and J. Li, Science 346, 1344 (2014).

[44] Y. G. Yao, L. Kleinman, A. H. MacDonald, J. Sinova, T. Jungwirth, D.-S. Wang, E. Wang, and Q. Niu, Phys. Rev. Lett. 92, 037204 (2004).

[45] Y. Yang, Z. Xu, L. Sheng, B. Wang, D. Y. Xing, and D. N. Sheng, Phys. Rev. Lett. 107, 066602 (2011).

[46] E. Prodan, Phys. Rev. B 83, 195119 (2011).

[47] H. Zhang, F. Freimuth, G. Bihlmayer, S. Blügel, and Y. Mokrousov, Phys. Rev. B 86, 035104 (2012).

[48] L. A. Wray, S.-Y. Xu, Y. Xia, D. Hsieh, A. V. Fedorov, Y. S. Hor, R. J. Cava, A. Bansil, H. Lin, and M. Z. Hasan, Nat. Phys. 7, 32 (2011).

[49] T. Cao, G. Wang, W. Han, H. Ye, C. Zhu, J. Shi, Q. Niu, P. Tan, E. Wang, B. Liu, and J. Feng, Nat. Commun. 3, 887 (2012).

[50] K. Yang, W. Setyawan, S. Wang, M. B. Nardelli, and S. Curtarolo, Nat. Mater. 11, 614 (2012). 